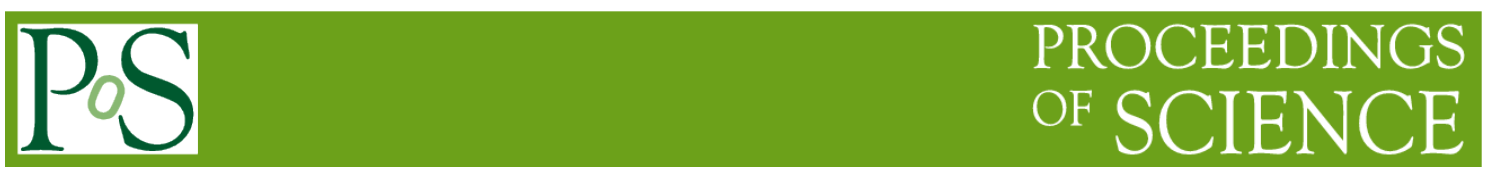

\title{
Soft probes, heavy flavour and quarkonia production in heavy-ion collisions with CMS
}

\section{Sergey Petrushanko* (for the CMS Collaboration)}

Skobeltsyn Institute of Nuclear Physics, M. V. Lomonosov Moscow State University,

Leninskie gory, Moscow 119991, Russian Federation

E-mail: Serguei.Petrouchanko@cern.ch

We review most recent the CMS detector results on soft probes, heavy flavour and quarkonia production in different types of heavy-ion collisions at the LHC.

40th International Conference on High Energy physics - ICHEP2020

July 28 - August 6, 2020

Prague, Czech Republic (virtual meeting)

"Speaker 


\section{Introduction}

The experiments at the Large Hadron Collider (LHC) have reported new results in pp, $\mathrm{pPb}$, $\mathrm{PbPb}$, and $\mathrm{XeXe}$ collisions over the past years.

The Compact Muon Solenoid (CMS) is a general purpose detector very well suited for the study of heavy-ion collisions, and its detailed description can be found in [1]. First heavy-ion $\mathrm{PbPb}$ collisions were recorded with the CMS detector during the night on 7 November 2010 at 00:27 Geneva time, and after almost ten years of operation the CMS Collaboration has published exactly one hundred heavy-ion papers and also has shown a number of preliminary results. All of the CMS heavy-ion papers can be found freely at the CMS website [2].

These proceedings briefly review the most interesting recent results from the CMS Collaboration on soft probes, heavy flavour and quarkonia productions in different types of heavy-ion collisions at the LHC.

\section{Soft probes in heavy-ion collisions with the CMS detector}

Soft probes, i.e. particles with low energy, allow us to probe the global properties of the system formed in heavy-ion collisions.

The pseudorapidity distributions of charged hadrons in $\mathrm{pPb}$ collisions at nucleon-nucleon center-of-mass energies $\sqrt{s_{N N}}=5.02$ and $8.16 \mathrm{TeV}$ were reported in [3]. The particle densities per participant nucleon are compared to similar measurements in the other experiments. $\quad \sqrt{s_{N N}}$-dependences for pp, pA, and AA collisions follow power laws.

The almost hermetic coverage of the CMS detector was used to measure the distribution of transverse energy over 13.2 units of pseudorapidity $\eta$. As an example, the transverse energy density versus $\eta$ for $\mathrm{pPb}$ collisions at $\sqrt{s_{N N}}=5.02 \mathrm{TeV}$ is shown in Figure 1 [4].

Long-range $(2<|\Delta \eta|<4)$, near-side (azimuthal angle $\Delta \varphi \approx 0$ ) angular correlations ("ridge"-effect) were observed with the CMS detector in high multiplicity pp [5] and pPb [6] collisions (as well as in $\mathrm{PbPb}$ collisions [7]). One of the possible interpretations of this effect is the interplay of independent elliptical and triangular harmonics [8], as it was shown with simulation with the HYDJET++ Monte-Carlo generator [9].

Measurements of two- and multi-particle angular correlations in pp collisions were presented [10] as a function of charged-particle multiplicity and were compared with the same measurements in $\mathrm{pPb}$ and $\mathrm{PbPb}$ collisions [11]. The elliptic azimuthal anisotropy coefficient $v_{2}$ extracted from long-range two-particle correlations is similar for $\mathrm{pp}$ and $\mathrm{pPb}$. This fact could be the manifestation of the collective origin for the observed long-range correlations in highmultiplicity pp collisions.

Azimuthal correlations of charged particles in XeXe collisions at $\sqrt{s_{N N}}=5.44 \mathrm{TeV}$ were studied [12]. The magnitude of the $v_{2}$ coefficients for XeXe collisions is larger than those found in $\mathrm{PbPb}$ collisions for the most central collisions. This is attributed to a larger fluctuation component in the lighter colliding system. Hydrodynamic models that consider the Xe nuclear deformation [13] better describe the $v_{2}[\mathrm{XeXe}] / v_{2}[\mathrm{PbPb}]$ ratio in central collisions than those assuming a spherical Xe shape. 
The mixed higher-order anisotropic flow and nonlinear response coefficients of charged particles are measured as a function of transverse momentum $p_{\mathrm{T}}$ and centrality in $\mathrm{PbPb}$ collisions at $\sqrt{s_{N N}}=2.76$ and $5.02 \mathrm{TeV}$ [14]. The results are compared with viscous hydrodynamic calculations using several different initial conditions, as well as microscopic transport model calculations. None of the models provides a simultaneous description of the mixed higher-order flow harmonics and nonlinear response coefficients.

The elliptic flow $v_{2}$ was measured for charm and strange hadrons in $\mathrm{pPb}$ collisions collected by the CMS experiment at $\sqrt{s_{N N}}=8.16 \mathrm{TeV}$ [15]. A significant positive $v_{2}$ signal from long-range azimuthal correlations is observed for all particle species in high-multiplicity $\mathrm{pPb}$ collisions. The results suggest that charm quarks have a smaller $v_{2}$ than lighter quarks, probably reflecting incomplete participation to the system collective motion. For $p_{\mathrm{T}}$ in the range of $2-5 \mathrm{GeV} / c$, the results suggest that $v_{2}$ for nonprompt $\mathrm{D}^{0}$ mesons are smaller than those for prompt $\mathrm{D}^{0}$ mesons [16].

Elliptic flow characterizing the azimuthal distribution of $\Upsilon(1 \mathrm{~S})$ and $\Upsilon(2 \mathrm{~S})$ mesons arising in $\mathrm{PbPb}$ collisions at $\sqrt{s_{N N}}=5.02 \mathrm{TeV}$ was studied [17]. In contrast to the $\mathrm{J} / \psi$ mesons, no azimuthal anisotropy was observed for the $\Upsilon$ mesons as shown in Figure 2.

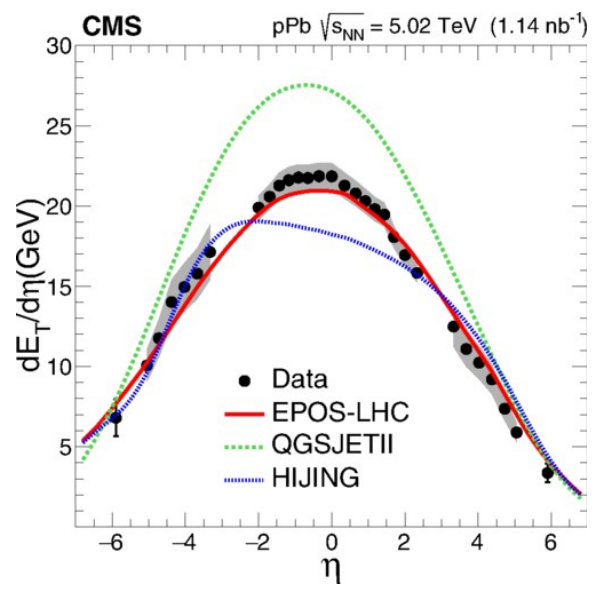

Figure 1. Transverse energy density versus pseudorapidity $\eta$ from minimum bias $\mathrm{pPb}$ collisions at $\sqrt{s_{N N}}=5.02 \mathrm{TeV}$ with $\mathrm{CMS}$ detector [4]. The proton is moving toward positive $\eta$. Predictions from event generators are also shown.

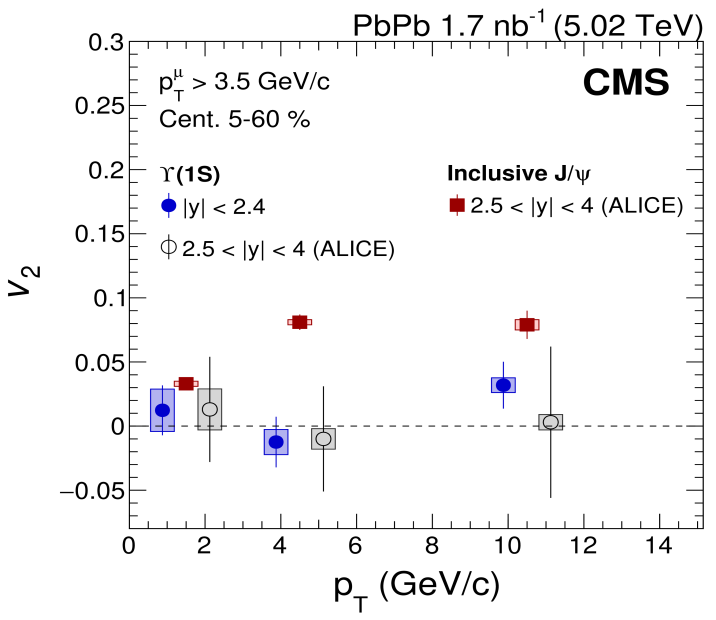

Figure 2. The $v_{2}$ for $\Upsilon(1 \mathrm{~S})$ mesons as a function of $p_{\mathrm{T}}$ in the rapidity range $|y|<2.4$ in $\mathrm{pPb}$ collisions at $\sqrt{s_{N N}}=5.02 \mathrm{TeV}$ with the CMS detector [17] (full circles) compared with the ALICE results [18] for $\Upsilon(1 \mathrm{~S})$ (open circles) and $\mathrm{J} / \psi$ (full squares) mesons measured in $2.5<|y|<4$.

\section{Heavy flavours and quarkonia in heavy-ion collisions with the CMS detector}

The cross sections for $\Upsilon(1 \mathrm{~S}), \Upsilon(2 \mathrm{~S})$, and $\Upsilon(3 \mathrm{~S})$ production in $\mathrm{PbPb}$ and pp collisions at $\sqrt{s_{N N}}=5.02 \mathrm{TeV}$ have been measured [19]. The nuclear modification factors, $R_{\mathrm{AA}}$, derived 
from the $\mathrm{PbPb}$-to-pp yield ratio for each state, are studied as a functions of meson rapidity and transverse momentum, as well as $\mathrm{PbPb}$ collision centrality. The yields of all three states are found to be significantly suppressed, and compatible with a sequential ordering of the suppression. No evidence of $\Upsilon(3 \mathrm{~S})$ signal in $\mathrm{PbPb}$ collisions was found.

Production cross sections of $\Upsilon(1 \mathrm{~S}), \Upsilon(2 \mathrm{~S})$, and $\Upsilon(3 \mathrm{~S})$ states produced in $\mathrm{pPb}$ collisions are reported using data collected by the CMS experiment at $\sqrt{s_{N N}}=5.02 \mathrm{TeV}$ [20]. All $\Upsilon$ states are found to be suppressed in $\mathrm{pPb}$ collisions compared to $\mathrm{pp}$ collisions with the same collision energy. The nuclear modification factors $R_{\mathrm{pPb}}$ show a sequential ordering, with $\Upsilon(1 \mathrm{~S})$ least suppressed and $\Upsilon(3 \mathrm{~S})$ most suppressed, indicating the presence of final-state modification of $\Upsilon$ states in $\mathrm{pPb}$ collisions. The suppression of $\Upsilon$ states in $\mathrm{PbPb}$ collisions is the largest when compared with $\mathrm{pPb}$ collisions.

The first evidence for $\chi_{\mathrm{cl}}(3872)$ production in relativistic heavy-ion collisions was observed with the CMS detector in $\mathrm{PbPb}$ collisions at $\sqrt{s_{N N}}=5.02 \mathrm{TeV}-$ see Figure 3 [21]. The results provide a unique experimental input to the theory, towards elucidating the production mechanism and the nature of $\chi_{\mathrm{cl}}(3872)$.

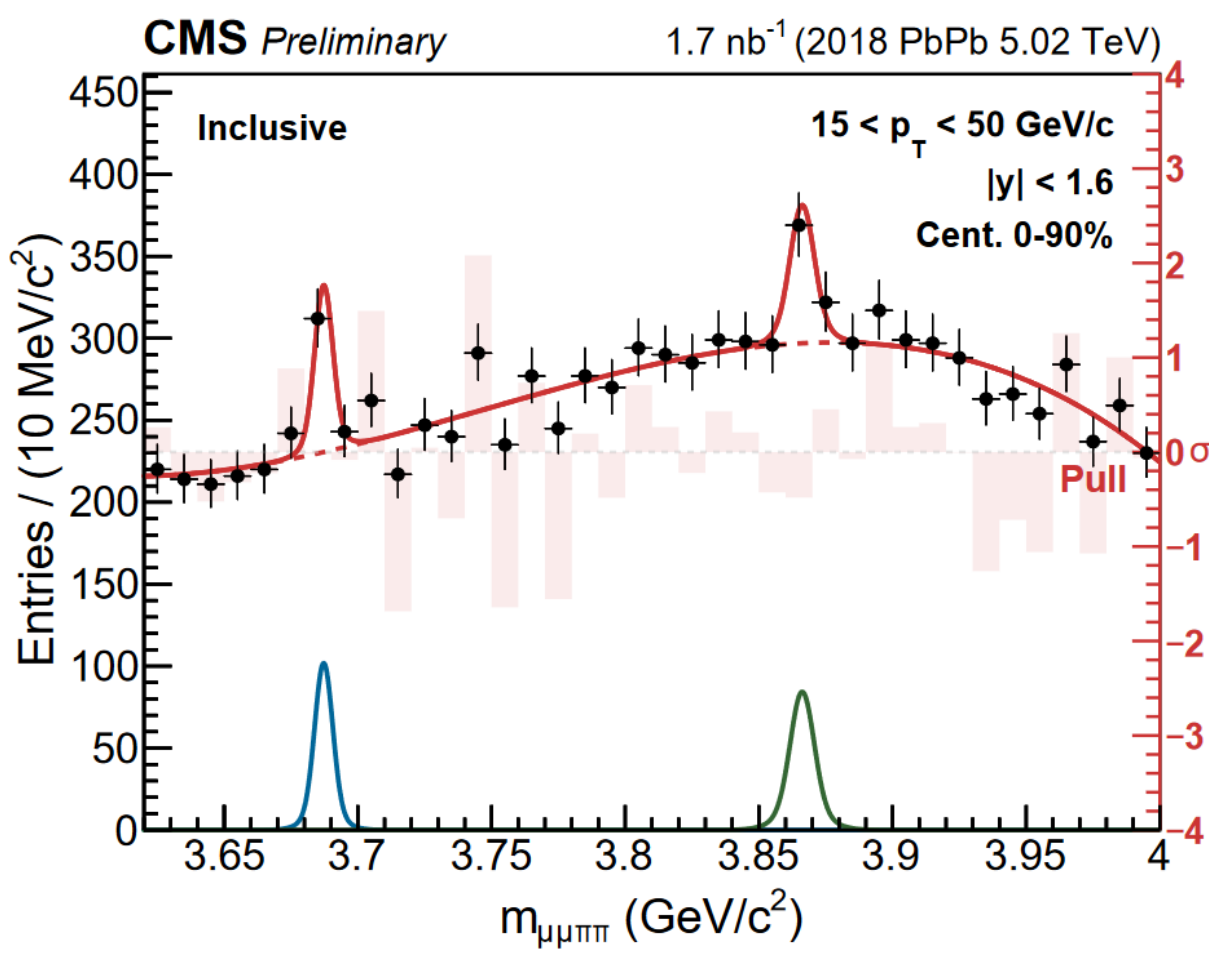

Figure 3. Distribution of invariant mass of $\mu \mu \pi \pi$ in $\mathrm{PbPb}$ collisions at $\sqrt{s_{N N}}=5.02 \mathrm{TeV}$ [21]. Events with centrality $0-90 \%$ are selected. The red line presents the unbinned maximumlikelihood fit result. The signal components of $\psi(2 S)$ and $\chi_{\mathrm{cl}}(3872)$ are shown by the blue and the green line respectively. The pull distribution is represented by the red boxes.

The $\mathrm{B}_{\mathrm{s}}^{0}$ and $\mathrm{B}^{+}$meson production cross sections are measured in $\mathrm{PbPb}$ collisions at $\sqrt{s_{N N}}=5.02 \mathrm{TeV}$ [22]. The $\mathrm{B}_{\mathrm{s}}^{0}$ meson is observed with a statistical significance in excess of 5 standard deviations for the first time in nucleus-nucleus collisions. 
First-ever evidence for the production of top quarks in nucleus-nucleus collisions, using $\mathrm{PbPb}$ collision data at $\sqrt{s_{N N}}=5.02 \mathrm{TeV}$ was presented by the CMS experiment [23]. Two methods are used to measure the cross section for top quark pair production via the decays into charged leptons (electrons or muons) and bottom quarks. The measured cross sections are compatible with expectations from scaled pp collision data and QCD predictions.

\section{Conclusions}

Experimental studies of heavy-ion collisions with the CMS detector at the LHC during the first two data taking periods (Run 1 and Run 2) already led to very interesting results in physics of hadronic matter under extreme conditions in terms of energy density and temperature: a number of physical observables have been measured at the new energies, various manifestations of collective effects were found, the suppression of the yields of bound states of heavy quarks is studied. We expect that in the course of the next operating periods of the LHC, Run 3 starting at the end of 2021, and Run 4 from 2026 onwards, we will be able to obtain very interesting new information about quark-gluon matter with the help of a significantly upgraded CMS detector and the expected record energy and luminosity of the accelerator.

\section{Acknowledgments}

I would like to thank all of the members of the CMS Collaboration for providing the materials for my talk and these proceedings. Also many thanks (Děkuji mnohokrát! Большое спасибо!) to the Organizers of the "virtual" 40th International Conference on High Energy Physics (ICHEP 2020) for the possibility to present the talk and for the nice management of the conference. The work was supported in part by Russian Foundation for Basic Research (grant 18-02-00155).

\section{References}

[1] CMS Collaboration, The CMS Experiment at the CERN LHC, JINST 3 (2008) S08004.

[2] http://cms-results.web.cern.ch/cms-results/publicresults/publications/HIN/

[3] CMS Collaboration, Pseudorapidity distributions of charged hadrons in proton-lead collisions at $\sqrt{s_{N N}}=5.02$ and $8.16 \mathrm{TeV}$, JHEP 01 (2018) 045.

[4] CMS Collaboration, Centrality and pseudorapidity dependence of the transverse energy density in pPb collisions at $\sqrt{S_{N N}}=5.02 \mathrm{TeV}$, Phys. Rev. C $100 \quad$ (2019) 024902.

[5] CMS Collaboration, Observation of long-range, near-side angular correlations in proton-proton collisions at the LHC, JHEP 09 (2010) 091.

[6] CMS Collaboration, Observation of long-range, near-side angular correlations in pPb collisions at the LHC, Phys. Lett. B 718 (2013) 795. 
[7] CMS Collaboration, Long-range and short-range dihadron angular correlations in central $\mathrm{PbPb}$ collisions at $\sqrt{s_{N N}}=2.76 \mathrm{TeV}$, JHEP 07 (2011) 076.

[8] G.Eyyubova, V.L. Korotkikh, I.P. Lokhtin, S.V. Petrushanko, A.M. Snigirev, L. Bravina, and E.E. Zabrodin, Angular dihadron correlations as an interplay between elliptic and triangular flows, Phys. Rev. C 91 (2015) 064907.

[9] I.P. Lokhtin, L.V. Malinina, S.V. Petrushanko, A.M. Snigirev, I. Arsene, and K. Tywoniuk, Heavy ion event generator HYDJET++ (HYDrodynamics plus JETs), Comp. Phys . Comm . 180 (2009) 779.

[10] CMS Collaboration, Evidence for collectivity in pp collisions at the LHC, Phys. Lett. B 765 (2017) 193.

[11] CMS Collaboration, Multiplicity and transverse momentum dependence of two- and four-particle correlations in pPb and PbPb collisions, Phys. Lett. B 724 (2013) 213.

[12] CMS Collaboration, Charged-particle angular correlations in XeXe collisions at $\sqrt{s_{N N}}=5.44$ TeV, Phys. Rev. C 100 (2019) 044902.

[13] G. Giacalone, J. Noronha-Hostler, M. Luzum, and J.-Y. Ollitrault, Hydrodynamic predictions for 5.44 TeVXe+Xe collisions, Phys. Rev. C 97 (2018) 034904.

[14] CMS Collaboration, Mixed higher-order anisotropic flow and nonlinear response coefficients of charged particles in PbPb collisions at $\sqrt{s_{N N}}=2.76$ and $5.02 \mathrm{TeV}$, EPJ C 80 (2020) 534.

[15] CMS Collaboration, Elliptic flow of charm and strange hadrons in high-multiplicity $p+P b$ collisions at $\sqrt{s_{N N}}=8.16 \mathrm{TeV}$, Phys. Rev. Lett. 121 (2018) 082301.

[16] CMS Collaboration, Studies of charm and beauty hadron long-range correlations in pp and $\mathrm{pPb}$ collisions at LHC energies, Phys. Lett. B 813 (2021) 136036.

[17] CMS Collaboration, Measurement of the azimuthal anisotropy of $Y(1 S)$ and $Y(2 S)$ mesons in PbPb collisions at $\sqrt{s_{N N}}=5.02 \mathrm{TeV}$, arXiv:2006.07707 [hep-ex].

[18] ALICE Collaboration, Measurement of $\mathrm{Y}(1 \mathrm{~S})$ elliptic flow at forward rapidity in $\mathrm{Pb}$-Pb collisions at $\sqrt{s_{N N}}=5.02 \mathrm{TeV}$, Phys. Rev. Lett. 123 (2019) 192301.

[19] CMS Collaboration, Measurement of nuclear modification factors of $Y(1 S), Y(2 S)$, and $Y(3 S)$ mesons in PbPb collisions at $\sqrt{s_{N N}}=5.02 \mathrm{TeV}$, Phys. Lett. B 790 (2019) 270.

[20] CMS Collaboration, Nuclear modification of $Y$ states in pPb collisions at $\sqrt{s_{N N}}=5.02 \mathrm{TeV}$, CMS-PAS-HIN-18-005.

[21] CMS Collaboration, Evidence for $\chi_{c 1}(3872)$ in PbPb collisions and studies of its prompt production at $\sqrt{s_{N N}}=5.02 \mathrm{TeV}, \mathrm{CMS}-\mathrm{PAS}-\mathrm{HIN}-19-005$.

[22] CMS Collaboration, Measurement of $B_{s}^{0}$ and $B^{+}$meson yields in PbPb collisions at $\sqrt{s_{N N}}=$ 5.02 TeV, CMS-PAS-HIN-19-011.

[23] CMS Collaboration, Evidence for top quark production in nucleus-nucleus collisions, Phys. Rev. Lett. 125 (2020) 222001. 